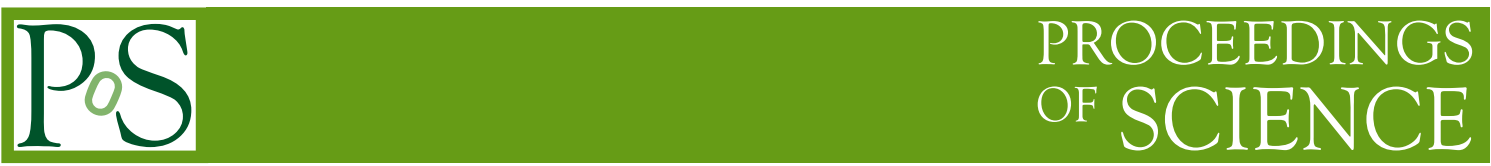

\title{
First Alignment of the Complete CMS Silicon Tracker
}

\author{
Jula Draeger* for the CMS collaboration \\ Institute of Experimental Physics, University of Hamburg, Hamburg, Germany \\ E-mail: jula.draeger@cern.ch
}

\begin{abstract}
We present the first results of the full CMS Silicon Tracker alignment based on several millions reconstructed tracks from the cosmic data taken during the commissioning runs with the detector in its final position. The all-silicon design of the CMS Tracker poses new challenges in aligning a complex system with 15148 silicon strip and 1440 silicon pixel modules. For optimal trackparameter resolution, the position and orientation of its modules need to be determined with a precision of several micrometers. For those modules well illuminated by cosmic ray particles, the ultimate precision has been achieved with data from the silicon modules traversed in-situ by charged muons used in combination with survey measurements. The achieved resolution in all five track parameters is controlled with data-driven validation of the track parameter measurements near the interaction region, and tested against prediction with detailed detector simulation.
\end{abstract}

9th International Conference on Large Scale Applications and Radiation Hardness of Semiconductor Detectors, RD09

30 Sep-2 Oct 2009

Florence, Tuscany (Italy)

\footnotetext{
* Speaker.
} 


\section{Contents}

1. Motivation and Strategy of Track-based Alignment 2

2. Validation of the Alignment Results

\section{Motivation and Strategy of Track-based Alignment}

As most physics analyses require a high precision of track parameters and the CMS tracker [1] consists of 1440 silicon pixel and 15148 silicon strip modules, their alignment is a challenging task and an important aspect in terms of a good detector understanding. During a continuous datataking exercise in October and November 2008, known as Cosmic Run At Four Tesla (CRAFT), 270 million cosmic-ray-triggered events were recorded, of which about 3 million provided useful tracks for the alignment procedure. The solenoid was operated at its nominal axial field strength of 3.8 T. The operating temperature of the tracker during the CRAFT data-taking period was stable at around 11 degrees Celsius. Excellent performance of the tracking system has been achieved with both the silicon strip [2] and silicon pixel [3] components.

The CMS pixel tracker consists of two sub-detectors, the barrel (BPIX) and the two endcaps in the forward regions (FPIX), shown in Fig. 1. The pixel modules provide two-dimensional measurements of the hit positions in the module planes, which effectively translate into three-dimensional measurements in space. The silicon strip detector is composed of four sub-detectors: the Tracker

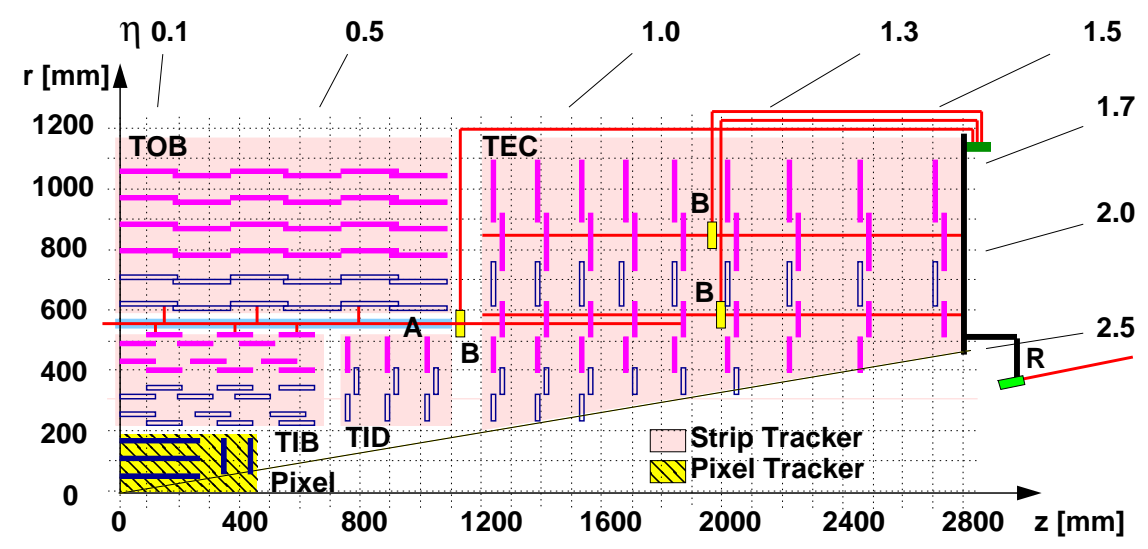

Figure 1: A quarter of the CMS silicon tracker in an $r z$ view. Single-sided silicon strip module positions are indicated as solid light (purple) lines, double-sided strip modules as open (blue) lines, and pixel modules as solid dark (blue) lines. Also shown are the paths of the laser rays (R), the beam splitters (B), and the alignment tubes (A) of the Laser Alignment System which is not discussed here.

Inner and Outer Barrels (TIB and TOB), the Tracker Inner Disks (TID), and the Tracker Endcaps 
(TEC). All sub-detectors are concentrically arranged around the nominal beam axis. The two inner layers of both the TIB and TOB, the two inner rings of the TID, and the first, second, and fifth rings of the TEC are equipped with double-sided modules, indicated in blue in Fig. 1; all other positions have single-sided modules [1]. Although the double-sided modules are glued together, the alignment was performed separately for the two module units. The goal of the track-based alignment procedures is to determine the module positions from a large sample of reconstructed charged particle trajectories by solving an optimization problem that can be formulated in the context of linear least squares.

A local right-handed coordinate system is defined for each module with the origin at the geometric center of the active area of the module. As illustrated in Fig. \& the $u$-axis is defined along the more precisely measured coordinate of the module (typically along the azimuthal direction in the global system), the $v$-axis orthogonal to the $u$-axis and in the module plane, pointing away from the readout electronics, and the $w$-axis normal to the module plane. When double-sided modules are considered as a single entity, the coordinate system is referenced to the $r \phi$ module.

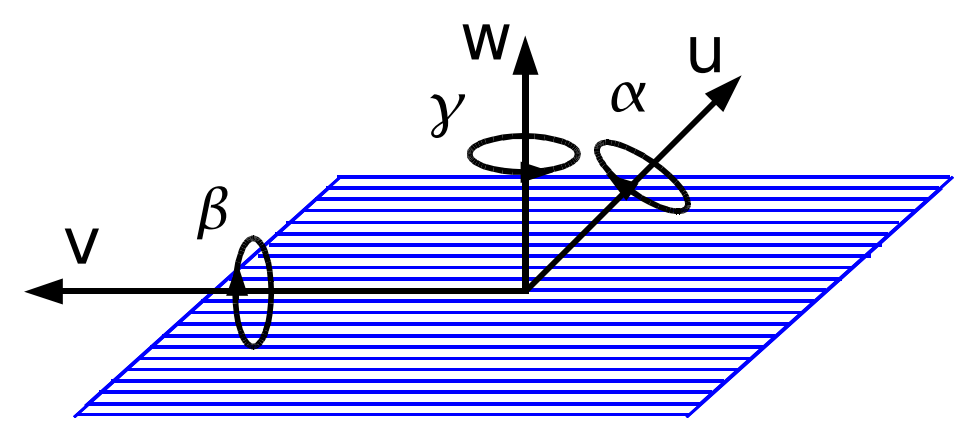

Figure 2: Illustration of the module local coordinates $u, v, w$ and the corresponding rotations $\alpha, \beta, \gamma$ for a single-sided strip module.

Module position corrections ("alignment parameters") $\mathbf{p}$ are determined by minimizing an objective function

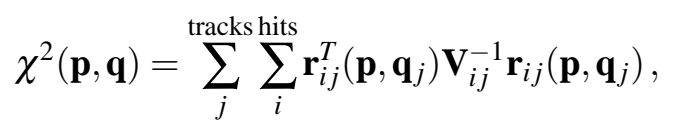

which can be expressed as the sum over all hits $i$ on all tracks $j$ with track parameters $\mathbf{q}_{j}$, assuming negligible correlations between hits. Track residuals $\mathbf{r}_{i j}=\mathbf{m}_{i j}-\mathbf{f}_{i j}\left(\mathbf{p}, \mathbf{q}_{j}\right)$ are defined as the difference between the measured hit position $\mathbf{m}_{i j}$ and the trajectory impact point $\mathbf{f}_{i j}$ and $\mathbf{V}_{i j}$ is the corresponding covariance matrix.

Two statistical methods were employed to solve the alignment problem. Both of them were previously applied to the CMS silicon strip tracker alignment during stand-alone commissioning [4]. The global alignment algorithm ("Millepede II") [5] minimizes the $\chi^{2}$ function in Eq. (1.1) by taking into account track and alignment parameters simultaneously.

The local iterative algorithm ("Hits and Impact Points") [6] approximates Eq. (1.1) by assuming no track parameter $\mathbf{q}$ dependence and therefore ignores correlations between alignment parameters for different modules in one iteration. The trajectory impact point $\mathbf{f}_{\alpha j}$ is recalculated for each hit, removing the hit under consideration from the track fit. The track parameters and correlations 
between different modules are taken into account through iterations of the minimization procedure and refitting the tracks with new alignment constants after each iteration. Furthermore, it permits the inclusion of survey measurements in the formalism of Eq. (1.1], as described in Ref. [7]. Contrary to the global method, which does not take into account the effects of material in the tracker and assumes a simple helical trajectory for charged particles, the local iterative method uses the full implementation of the Kalman filter track reconstruction algorithm adopted in CMS [2]]. Therefore it requires a large number of iterations and large computing resources to re fit the tracks in each iteration. The global method, instead, allows the determination of alignment parameters, properly accounting for the correlations among them, in a single step. After verifying that the two methods yielded consistent results, the final results were obtained by applying the two algorithms in sequence in order to take advantage of their complementary strengths.

\section{Validation of the Alignment Results}

The validation of the achieved alignment corrections was performed using different techniques, starting from the monitoring of low level quantities that are minimized by the alignment algorithms, up to the validation of higher level quantities like the track parameters itself. The tracks used for the following alignment validation have at least 8 hits, two of them on a $2 \mathrm{~d}$ module and the transverse momentum is required to be larger than $4 \mathrm{GeV}$. Fig. B 3 shows the global $\chi^{2} / \mathrm{ndf}$ and the residual distribution for the Tracker Outer Barrel which is dominated by random effects like the track extrapolation uncertainties due to multiple scattering and the hit position reconstruction uncertainties. As misalignment leads to systematic shifts of the residual, a more appropriate way to measure the alignment precision is to take the distribution of the median $\mu_{1 / 2}$ of the residual (DMR) shown for the Tracker Outer Barrel and the Pixel Barrel in Fig. 4.
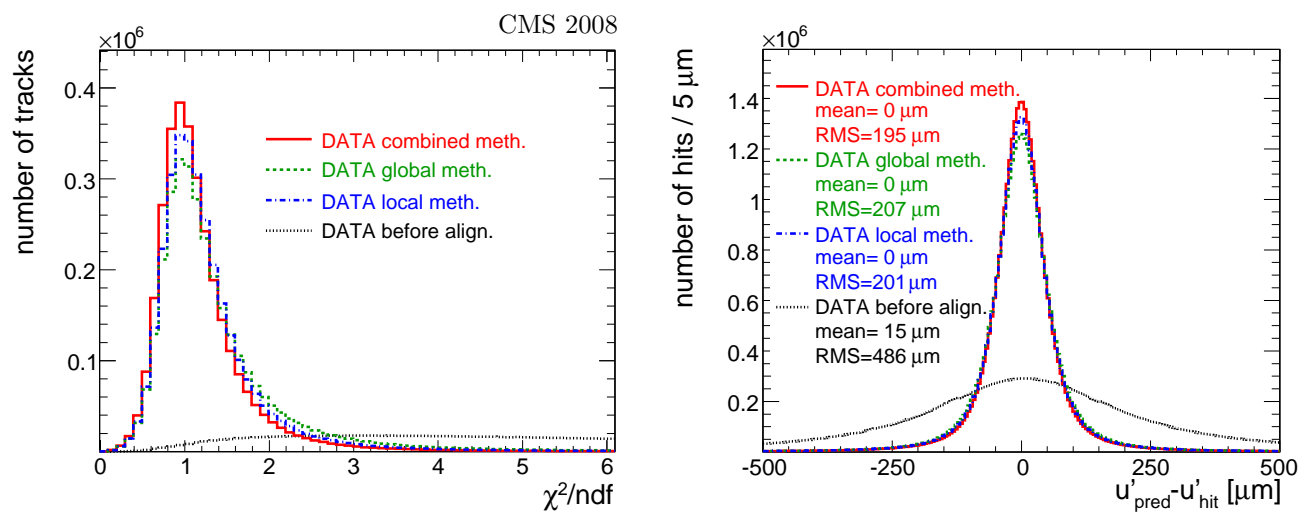

Figure 3: Distributions of the $\chi^{2} /$ ndf of the tracks and the residual distribution in the Tracker Outer Barrel before alignment (dotted line) and after alignment with the local (dashed-dotted line), global (dashed line), and combined (solid line) methods.

To check the statistical precision of the track-based alignment, a Monte Carlo (MC) simulation was performed in which module positions from the combined method obtained with data were used as the starting geometry in the MC alignment procedure. This approach in MC effectively models 

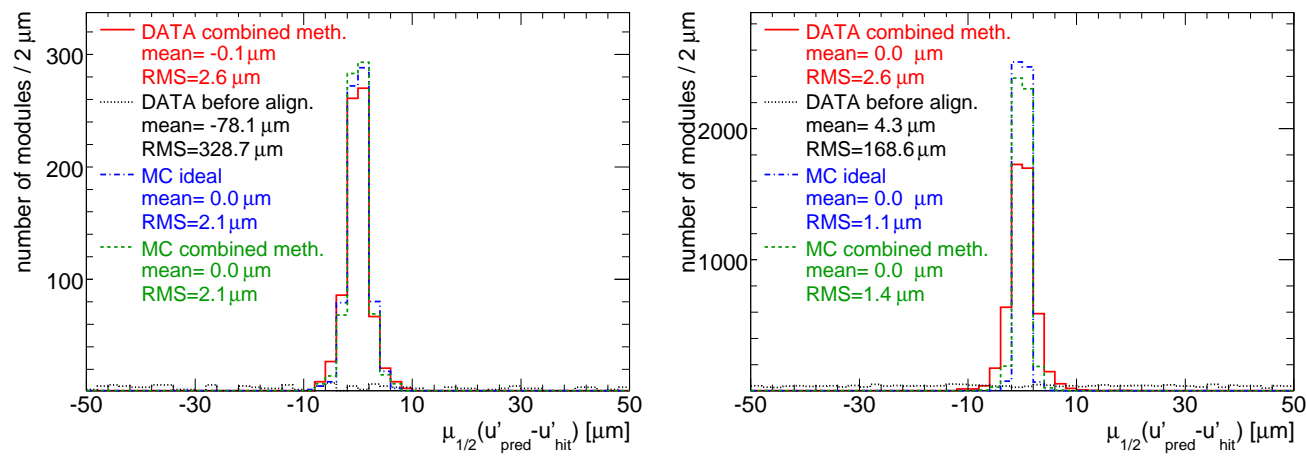

Figure 4: Distribution of $\mu_{1 / 2}$, the median of the residuals, for modules with more than 30 hits, shown for BPIX (left) and TOB (right). Shown are distributions before alignment (black dotted), after alignment with the combined method (red solid), combined method MC (green dash ed), and ideal MC (blue dash-dotted).

the situation in data prior to and during the alignment. The resulting DMRs are also shown in Fig. $\theta$ and the RMS values listed in Table 1. For comparison, the distributions obtained from the ideal MC simulation are presented in Fig. 7 as well.

Table 1: RMS of the distribution of the median of the residuals (DMR) in the $u^{\prime}$ and $v^{\prime}$ local coordinates for modules with more than 30 hits. The number of these modules compared to the total number of modules is stated in the last column. Four geometries are considered: those obtained with the three methods discussed in the text and the geometry before alignment. Results from simulations based on the combined alignment and ideal geometries are shown for comparison.

\begin{tabular}{|c|c|c|c|c|c|c|c|}
\hline & $\begin{array}{c}\text { before align. } \\
{[\mu \mathrm{m}]}\end{array}$ & $\begin{array}{c}\text { global } \\
{[\mu \mathrm{m}]}\end{array}$ & $\begin{array}{c}\text { local } \\
{[\mu \mathrm{m}]}\end{array}$ & $\begin{array}{c}\text { combined } \\
{[\mu \mathrm{m}]}\end{array}$ & $\begin{array}{c}\text { combined } \\
\mathrm{MC}[\mu \mathrm{m}]\end{array}$ & $\begin{array}{c}\text { ideal } \\
\mathrm{MC}[\mu \mathrm{m}]\end{array}$ & $\begin{array}{c}\text { modules } \\
>30 \text { hits }\end{array}$ \\
\hline BPIX $\left(u^{\prime}\right)$ & 328.7 & 7.5 & 3.0 & 2.6 & 2.1 & 2.1 & $757 / 768$ \\
BPIX $\left(v^{\prime}\right)$ & 274.1 & 6.9 & 13.4 & 4.0 & 2.5 & 2.4 & \\
FPIX $\left(u^{\prime}\right)$ & 389.0 & 23.5 & 26.5 & 13.1 & 12.0 & 9.4 & $393 / 672$ \\
FPIX $\left(v^{\prime}\right)$ & 385.8 & 20.0 & 23.9 & 13.9 & 11.6 & 9.3 & \\
TIB $\left(u^{\prime}\right)$ & 712.2 & 4.9 & 7.1 & 2.5 & 1.2 & 1.1 & $2623 / 2724$ \\
TOB $\left(u^{\prime}\right)$ & 168.6 & 5.7 & 3.5 & 2.6 & 1.4 & 1.1 & $5129 / 5208$ \\
TID $\left(u^{\prime}\right)$ & 295.0 & 7.0 & 6.9 & 3.3 & 2.4 & 1.6 & $807 / 816$ \\
TEC $\left(u^{\prime}\right)$ & 216.9 & 25.0 & 10.4 & 7.4 & 4.6 & 2.5 & $6318 / 6400$ \\
\hline
\end{tabular}

A further method to monitor and validate the results of the alignment is to use the hits from tracks passing through regions where modules overlap within a layer of the tracker. This method, described in detail in Ref. [8], is also used to measure the hit resolution of the sensors [2]. In this method, the difference in residual values for the two measurements in the overlapping modules is compared. Deviations between the reconstructed hits and the predicted positions allow an assessment of the relative alignment between two adjacent modules as shown in Fig. 5. For the TIB the RMS value of the mean of the distributions of the relative shift between overlapping module pairs, 
scaled by $1 / \sqrt{2}$ to account for the two independent measurements, decreases from $264 \mu \mathrm{m}$ without alignment to $7 \mu \mathrm{m}$ after the alignment procedure using the combined method.

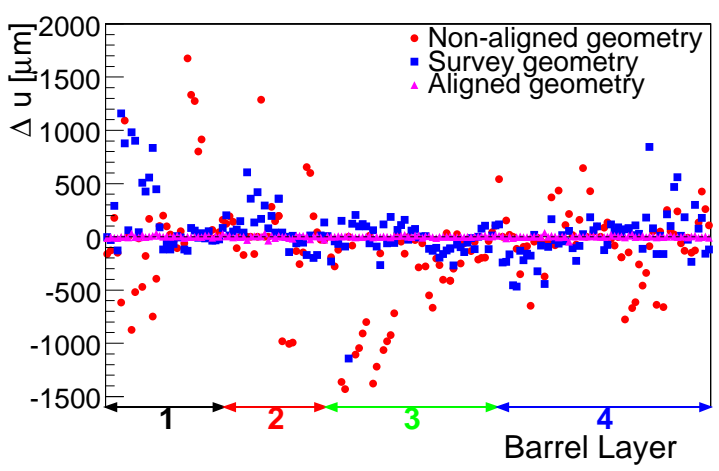

Figure 5: Relative shift between module pairs in the local $u$-coordinate in the TIB. Only modules in the slice $80^{\circ}<\phi<100^{\circ}$ are shown before alignment (red dots), including survey measurements (blue squares) and after alignment (purple triangles).

To validate the track parameter resolutions an independent reconstruction of the upper and the lower part of cosmic ray tracks can be used to compare the two sets of resulting track parameters at the point of closest approach to the nominal beamline. Both the upper and lower track segments were required to have at least three pixel hits to mimic the topology of collision tracks.

Fig. 6 shows the difference between upper and lower portions of tracks for all five track parameters. There is significant improvement due to tracker alignment, with good agreement between data and MC simulations. The normalized distributions in Fig. 6 also show that the error estimates on the track parameters are in good agreement with predictions from MC simulations. To estimate the remaining systematic misalignment which only effects the $\chi^{2}$ weakly and is thus referred to as 'weak mode', a set of systematically misaligned geometries was added on top of the alignment geometry and the alignment procedure was repeated, using the same strategy and dataset as before. Fig. 7 shows the detector geometry for a systematic layer rotation and an expansion in the global zdirection. Although the $\chi^{2}$-distribution can be recovered in both cases, the resulting geometry only shows a slight recovery in case of the layer rotation misalignment scenario and nearly no changes for the z-expansion. Systematic misalignment thus remains a challenge and shows the need for more data and especially complementary datasets for example from collision data.

The author would like to thank her colleagues from the CMS experiment, and especially from the tracker alignment group, for their collaboration and excellent team work which was leading to the presented results, the BMBF and Terascale Alliance for support and the conference organizers for their hospitality. 

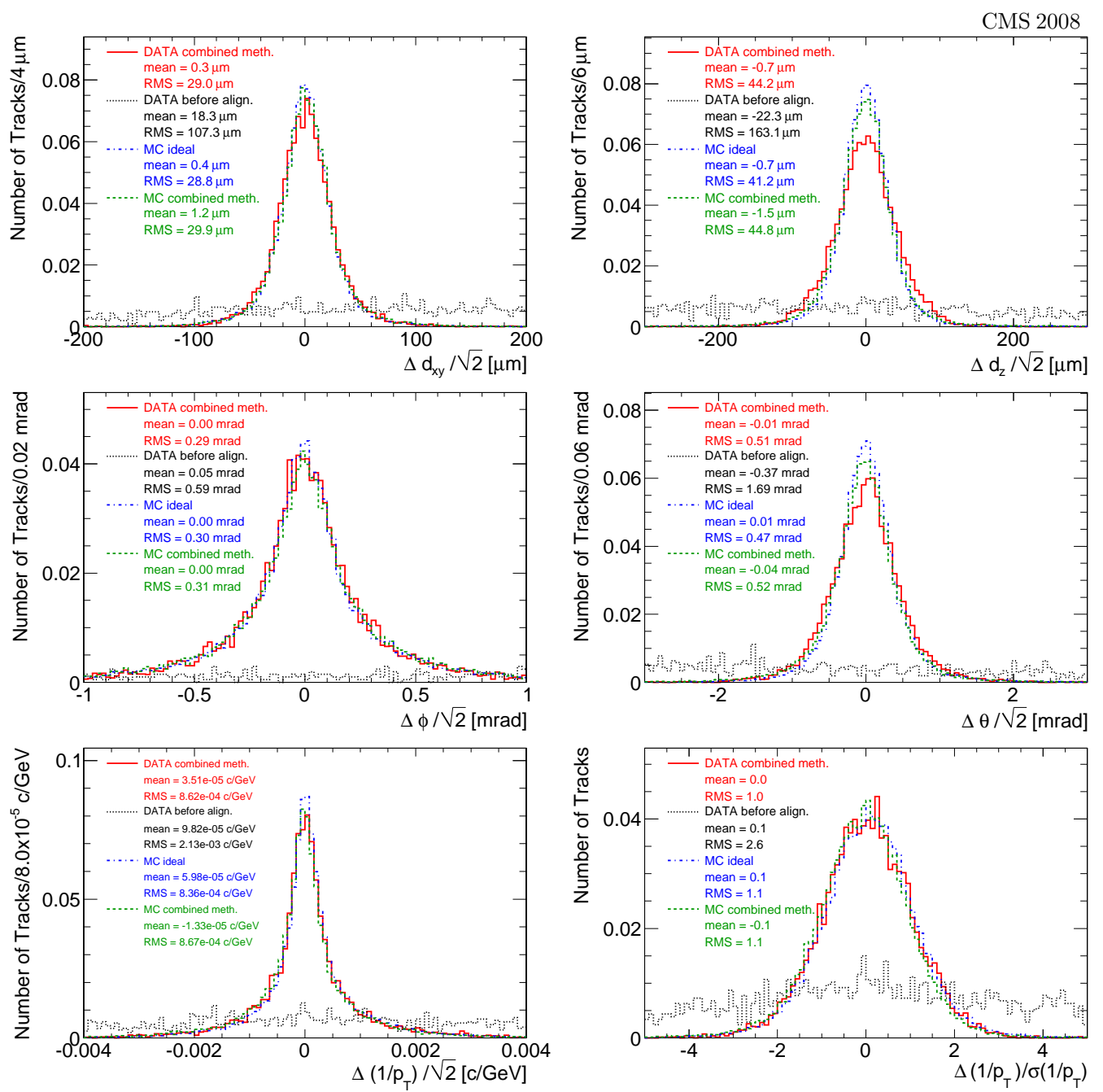

Figure 6: Differences between upper and lower track segment parameters measured at the point of closest approach to the beamline and scaled by $1 / \sqrt{2}$. Distributions are shown for the distance of closest approach in the transverse direction $d_{x y}$ (top left), the same in the longitudinal direction $d_{z}$ (top right), the track azimuthal angle $\phi$ (middle left), the track polar angle $\theta$ (middle right), and $1 / p_{T}$ (bottom left). The plot on the bottom right shows the $1 / p_{T}$ difference normalized to its error, that is $\left(1 / p_{T, 1}-1 / p_{T, 2}\right) / \sqrt{\sigma_{1 / p_{T}, 1}^{2}+\sigma_{1 / p_{T}, 2}^{2}}$. Results are shown for four geometries: data before alignment (black dotted lines), data with combined method alignment (red solid), combined method MC (green dashed), and ideal MC (blue dash-dotted). 

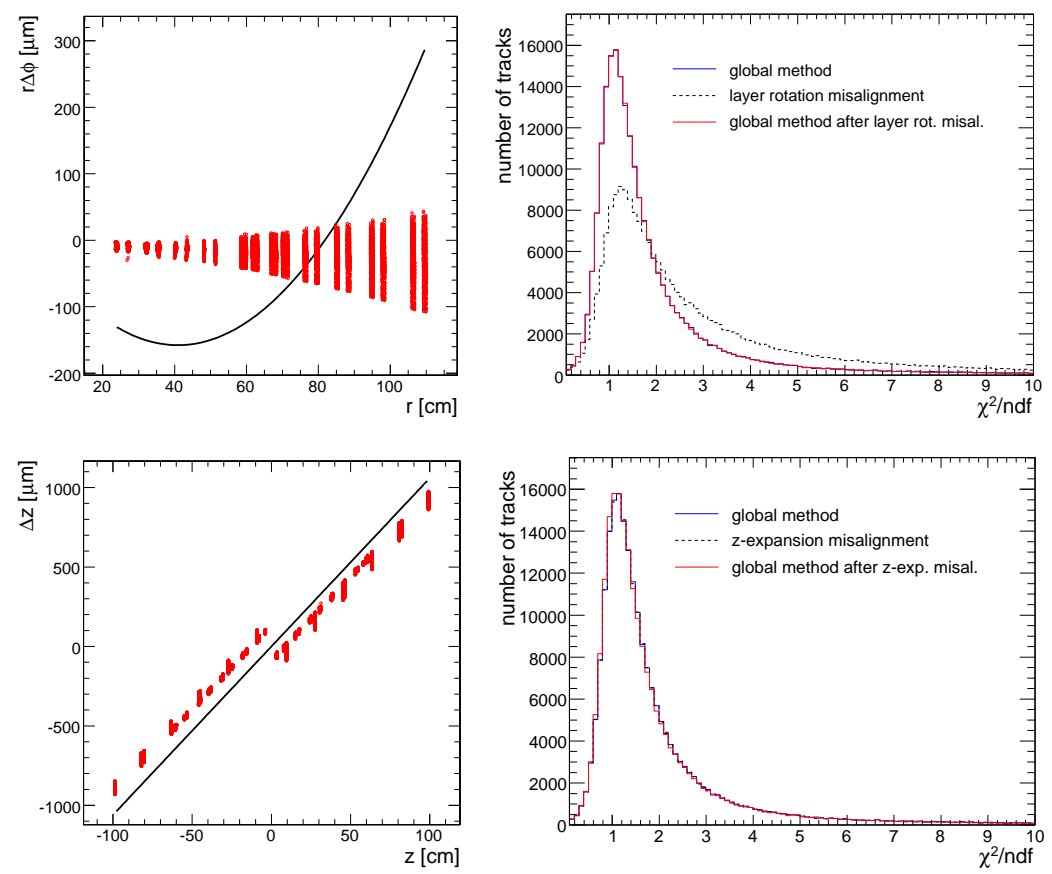

Figure 7: Comparison of the module positions in TIB and TOB with respect to the geometry obtained with the global method after applying systematic distortions (black solid lines) and after alignment (red dots),shown for layer rotation (left top row), and z-expansion (left bottom row) weak modes. The plots on the right show the distributions of the corresponding track $\chi^{2} /$ ndf after the alignment with the global method (blue solid line), after introducing the systematic misalignment (black dashed line), and after re-aligning (red solid line, below the blue solid line).

\section{References}

[1] R. Adolphi et al. [CMS Collaboration], JINST 3 S08004, 2008.

[2] CMS Collaboration, "The CMS Silicon Strip Tracker Operation and Performance with Cosmic Rays in 3.8 T Magnetic Field”CMS-CFT-09-002, to be submitted to JINST, 2009.

[3] CMS Collaboration, "Performance of the CMS Pixel detector with cosmic ray data ," CMS-CFT-09-001, to be submitted to JINST, 2009.

[4] CMS Tracker Collaboration, "Alignment of the CMS silicon strip tracker during stand-alone commissioning,” JINST 4 T07001, 2009.

[5] V. Blobel, "Software Alignment for Tracking Detectors," Nucl. Instr. Methods Phys. Res. A 566, 5, 2006.

[6] V. Karimäki, T. Lampén, and F.-P. Schilling, "The HIP Algorithm for Track Based Alignment and its Application to the CMS Pixel Detector," CMS NOTE-2006/018, 2006.

[7] D. N. Brown, A. V. Gritsan, Z. J. Guo, and D. A. Roberts, "Local Alignment of the BABAR Silicon Vertex Tracker,” Nucl. Instr. Methods Phys. Res. A 603, 467, 2009.

[8] CMS Tracker Collaboration, "Stand-alone cosmic muon reconstruction before installation of the CMS silicon strip tracker," JINST 4 P05004, 2009. 